\title{
COVID-19 Sex-Age Mortality Modeling: \\ A Use Case of Risk-Based Vaccine Prioritization
}

\author{
Vladimir Shapiro \\ Northeastern University, Boston, MA
}

\begin{abstract}
This research builds upon the previous publications claiming that the male sex population and both sex individuals of advanced age are more susceptible to COVID-19's risks. Relations between sex and age gradients are explored analytically based upon the proposed log-polynomial regression model of COVID-19 mortality. This model enables predicting mortality risk at any arbitrary age, as well as the derivation of several useful secondary metrics:

- $\quad$ Sex differential: a ratio of male-to-female death risks for a given age group.

- $\quad$ Age parity: age at which both sexes have an equal vulnerability.

- Age lag: the number of years to subtract from a male's age to match a female's death risk.

- Male equal risk age: male's age at which male's odds of dying from COVID-19 will equate female's given the cutoff age.
\end{abstract}

These metrics allow solving such practical problems as, e.g., prioritizing vaccine based on COVID-19 mortality risk associated with sex and age. Modeling techniques, refined in the paper, are by no means unique to COVID-19 and would apply to analyses of other diseases.

Keywords: COVID-19, vaccination, vaccine prioritization, age lag, male equal risk, parity age, sex differential, mortality, statistical modeling, demography 


\section{Introduction}

As the world plows through the subsequent waves of the COVID-19, scientists are busy analyzing the data and uncovering trends in attempts to minimize losses of human lives and disruptions to the economies.

In this paper, we will focus here on the most fundamental demographic characteristics such as age and sex to help us solving some practical problems. There have been numerous recent publications by e.g. (Demombynes 2020; Guilmoto 2020; Ng et al. 2020), supporting the following principal trends related to age and sex:

- $\quad$ Older populations are at higher COVID-19 death risks.

- Males are generally more vulnerable than females of the same age.

Progress has been made in researching the age gradients of COVID-19 deaths and quantification of the sex differential (SD) see e.g. (Guilmoto 2020). In other sources, the sex differential is referred to as $M / F$ (maleto-female ratio), see (Sobotka et al., 2020), or GMR (gender mortality ratio), see (Ng et al. 2020). The main research tool, employed by scientists, has been the linear regression applied to the stratified death counts to 100,000 of the general population of each sex on a logarithmic scale. However powerful, the linear regression has several limitations:

- It ignores the fact that the straight line rarely fits the stratified death counts on a logarithmic scale optimally; there is a subtle curvature that the data point outline would naturally have, see e.g., Figure 4 , which is not captured by a straight line. While limiting the regression to its linear form is convenient, it becomes a constraint when it comes to identifying trends and making more accurate predictions between the available data points (age groups) or beyond the last age groups of $80+$ or $90+$.

- It implies that all the age groups have equal death counts ("weights"), as the OLS (Ordinary Least Square) is used for fitting. The latter minimizes the sum of square differences between the observed and the fitted values thus ignoring the vastly higher relative weight of the older age death counts compared to the younger ones.

The research below is aiming at suggesting alternatives that are more adequate for the problems at hand.

Still, on the subject of the regression fit, there is another aspect that had attracted insufficient attention in the literature. Namely, selection of a "representative" value (age) for a given age group (bin/bucket), used in the fitting. We are discussing this subject in Chapter 2.4.

Upon having refined the modeling methodology, this research aims at further exploration of such metrics as age parity and age lag introduced in (Shapiro 2020), which are alternative metrics that describe different aspects of the sex-age mortality relations. The latter concept facilitates solving such a practical problem as the COVID-19 mortality risk-based vaccine prioritization of population strata based on age and sex in a fashion tailored to a given country, see Chapter 2.4.3. Out of several popular vaccine allocation criteria, see (Ferranna et al, 2021) ours is to decrease mortality as much as possible.

\section{Modeling}

The data, used in this research, originates from nine European countries and the US; all taken from the top of the list of the most severely affected by the COVID-19 with a total of over 900 thousand fatal outcomes as of March 2021 (INED 2021) making the dataset representative from both numerical and geographical diversity standpoints. The countries in question bear similarities from the population structure and other perspectives, and the identified trends are applicable to many of them as well as others not considered here.

By now a trove of national COVID-19 mortality datasets is publicly available. We defined the following criteria for dataset selection:

- Trustworthiness of a source.

- The minimum death count for a given country of at least $10 \mathrm{~K}$,

- Sufficient granularity (<=10-year intervals) ${ }_{2}-$ larger age intervals will not be suitable for robust 
modeling presenting too sparsely located data points.

- Half-open oldest age interval (histogram bin) to be $80+$ or older - death counts for this and all older ages are aggregated in the latter. Allowing the right-most interval to shift to even younger ages will risk making this interval dominate thus distorting the trend.

- Deaths are counted separately for each sex - we excluded some nation datasets that would aggregate both sexes in a single count.

Aggregated (binned) into 5- or 10-year age groups, all datasets have been procured from (INED 2021) and are based on the United Nations and national government agencies' input; all are available in open access. The only exception is the unique Czech Republic's single-year datasets sourced from (Czech Statistical Office 2019, MZČR Onemocnění Aktuálně 2021), also openly accessible. The countries under consideration have been through several pandemic waves; their fatality counts are numerous enough to manifest wellestablished trends.

\subsection{Log-Polynomial Model}

The polynomial regression in the logarithmic space, i.e. log-polynomial, see eq. (1), would be more accurate and thus suitable fit than the customary simple regression log-linear model e.g. (Demombynes 2020; Guilmoto 2020). We limited the polynomial to the 2nd degree to avoid the model overfitting the data:

$$
\log (y)=b_{0}+b_{1} x+b_{2} x^{2}
$$

where $y$ represents a death count for a given age group $x$ standardized (stratified) per 100,000 of given sex in the general population (INED 2021).

Instead of the conventional log-linear model, it is possible to fit Poisson regression models. As stated in (Cheng et al, 2016): "Poisson regression, a generalized linear model, is appropriate for modeling ... mortality rates". In its basic form the log-linear form of the Poisson regression has been used (Cheng et al, 2016, Tai and Noymer, 2018), which, just as the simple linear regression, is not complex enough to fit the non-linear nature of the COVID-19 death counts versus age distribution. There have been attempts to address that. An equation similar to Eq. 1 for the stratified death counts, are referred to as Poisson regression with e.g., "quadratic age effect" (Currie 2013) in the context of modeling mortality rates (death counts) as a function of age, or "age quadratic" (Andersen 2019, Atkinson et al 2008) when modeling lung cancer case numbers versus age.

\subsubsection{Ordinary (OLS) versus Weighted (WOLS) Regression}

Let us look closer at the mortality data plotted in Figure 1 (the same applies to the non-stratified data on e.g., Figure 7). The height of each bin there represents a number of fatalities (deaths) for a given age group. In other words, behind a data point at the age group of say 30-39 years, there are only a handful of deaths, whereas, behind the age group of say 80-89, there are thousands. For instance, in Germany both sex COVID-19 deaths for the age group of $80-89$ years are $46.4 \%$ of the total versus just $0.2 \%$ for $30-39$ years; the trend is very similar for the other countries observed such as for the Czech Republic, shown in Figure 1, where the death counts are proportional to the black circle diameters. This information would be implicitly ignored if we were to fit an OLS (Ordinary Least Square) regression, as put in "A set of unweighted normal equations for a least-squares solution assumes that the response variable of each equation is equally reliable and should be treated equally" (Shin 2013). OLS implies that each data point error (residual) contribution to the total OLS error is equal.

There are other, non-COVID-19, situations when some data points are more important than others, e.g. time series where the impact of the latest data may be valued higher (Wang, etc. 2020); data from some geographic areas may be more important than from others (Kala, etc. 2017), etc. As indicated in (Willett and Singer, 1988) "different weights are assigned to different observation in order to recognize that some 
observations are 'better 'or 'stronger ' than others". In our context of using regression fit for predicting COVID-19 mortality behavior for various age groups, the intuition suggests that those data points (histogram bins) that represent a large number of deaths are weighed "heavier" than others with a lot fewer deaths as in the case of younger ages.

In the context of COVID-19 death counts the lack of uniformity is more than obvious, see e.g. Figure 7(e,f) where stratified histograms are displayed for the Czech Republic; other country's trends are very similar. Another observation that stresses asymmetry between the data points, in this case, the groups of such, is that data points (age bins) of all three data point weights of "40-49", "50-59", "60-69" contain just around 3\% of the total death count; whereas another three data points of "70-79", "80-89", "90-99" - around 96\%. One can easily imagine that the OLS regression would unjustifiably boost the "40-69" range while suppressing the one of "70-99", causing the trend line slope tracking better in the younger ages where it matters less, and worse at older ages when it matters more, see Figure 1. The WOLS-fitted polynomials tend to have greater curvature than OLS ones.

For the rest of the paper, we will be applying the WOLS fitting technique ${ }^{1}$.

\section{Figure 1.}

WOLS (Weighted Ordinary Least Squares) versus OLS (Ordinary Least Square). Stratified death count as a weight factor for log-poly regression fit (male's data is on the top): (a) 10-year age bins, (b) 1-year age bins.

Ordinary vs Weighted Log-Poly Regression: Czech Republic (stratified per 100,000; clipped <100)

(a)

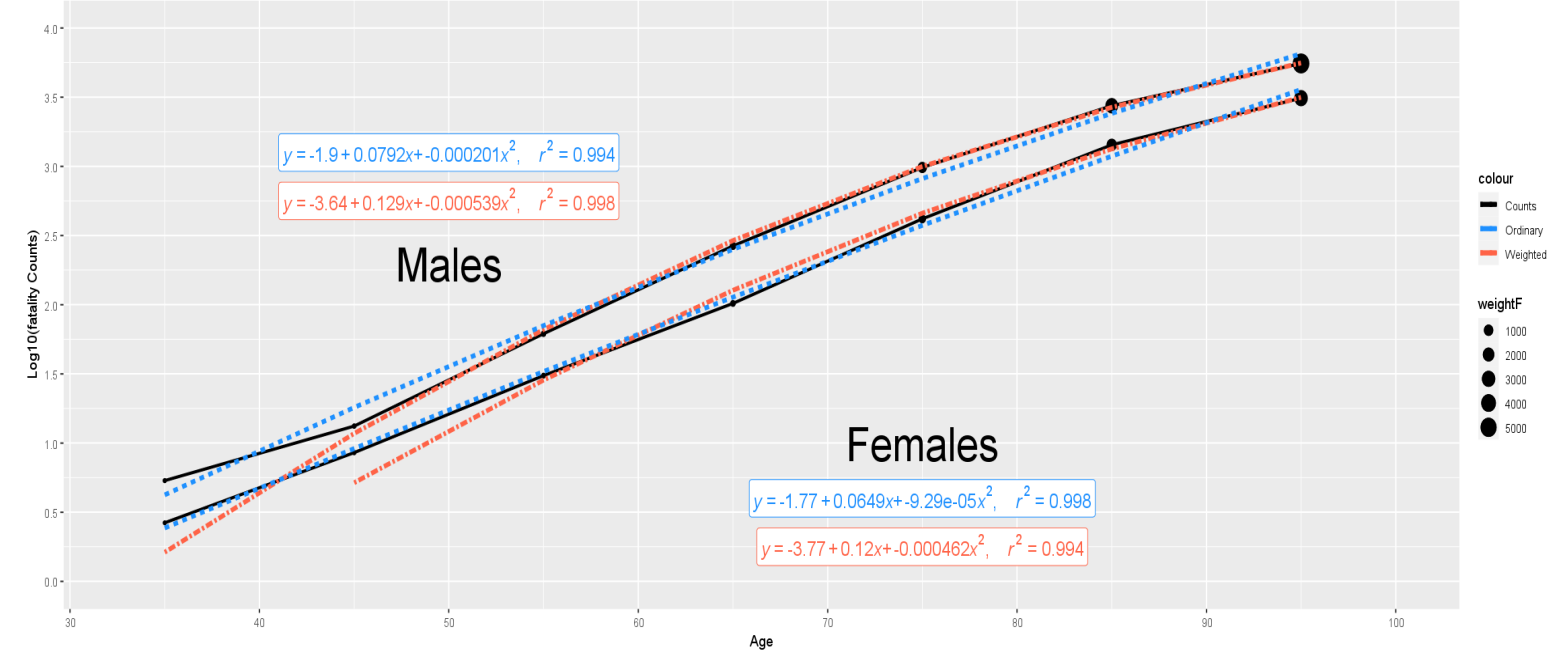

Ordinary vs Weighted Log-Poly Regression: Czech Republic (stratified per 100,000; clipped <100): 1-year bins

(b)

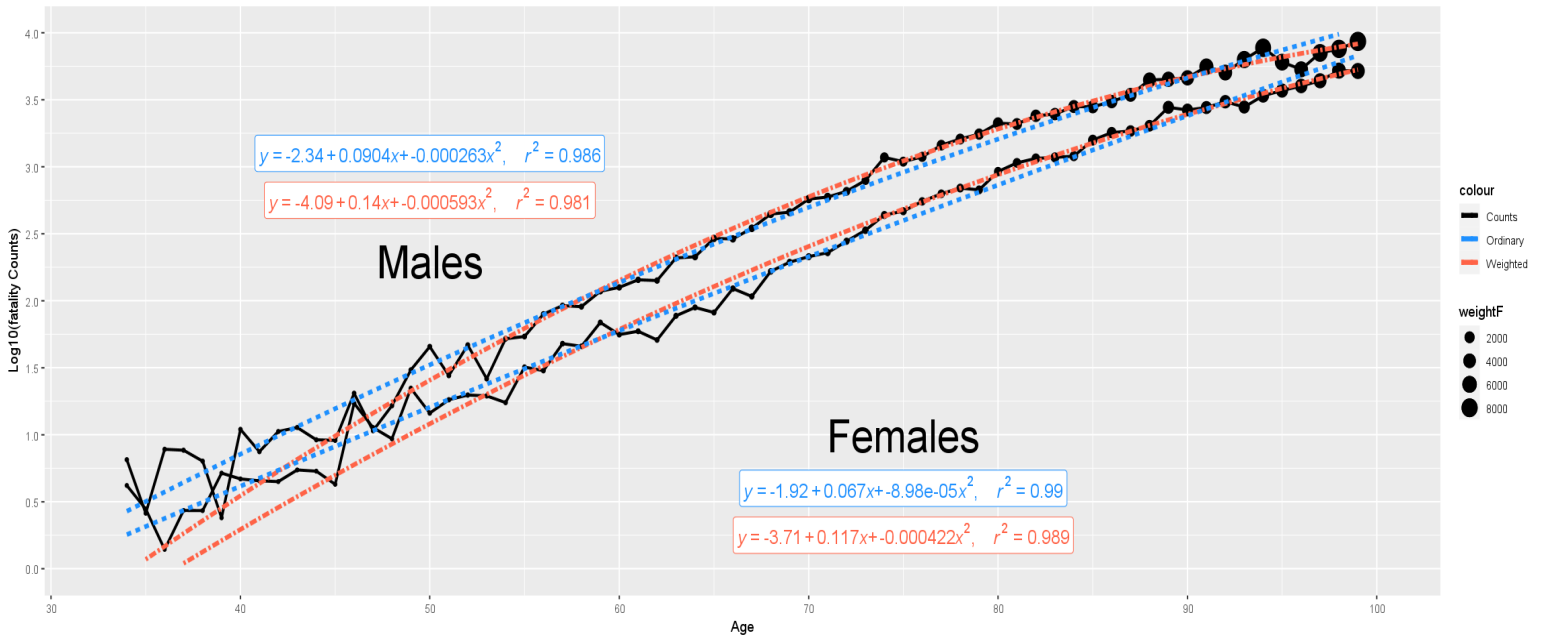

The OLS fit tracks closer to the low weight younger ages while the WOLS better fits the older ages data points in Figure 1. Note that the $R^{2}$ (the goodness of fit) may or may not improve as a result of applying the WOLS. As noted in (Willett and Singer, 1988) the focus should be "particularly the increased precision of

\footnotetext{
${ }^{1}$ Instead of WOLS, it would be possible to apply the weighting approach to the quadratic age Poisson regression model. We left this comparison out of scope assuming that superiority of WOLS over the Poisson log-linear regression models established in (Tai and Noymer, 2018) would hold if an extra quadratic term is addeqd to each model. However, this deserves further exploration.
} 
the estimates of $\beta$ " (regression coefficients) delivered by the WOLS, the method behind the calculation of the weighted regression, rather than the $R^{2}$ value.

As expected, it appears from Figure 1 that the weighted fit better follows the trend if the bin count (weight) is taken into account. It is not clear, however, how close the weighted regression on the 10-year age bin dataset approximates the single-year one. As mentioned above, the Czech Republic data are available in the single-year resolution along with the 10-year; the only exception was the "100+" age group which is a single consolidated data point. For the sake of simplicity, we excluded from consideration this " $100+$ " data point (more on the subject in Chapter 2.4) as well as the very young ages with insignificant death counts. We used that in Figure 2 below which shows the WFS fit to the 10-year bin size (in red), and single-year (in blue) to the highest resolution single-year. One can see that the 10 -year-based fit closely tracks the singleyear one including the major data points, shown with circles. Whatever the bin resolution we can notice that the WFS fit the heavy-weight old age data points nicely.

Figure 2.

WOLS versus OLS. Stratified death counts as weight factor on the 10-year versus single-year bin size. Ordinary (nonweighted regression on the 10 -year bin size is shown in black).

(a)
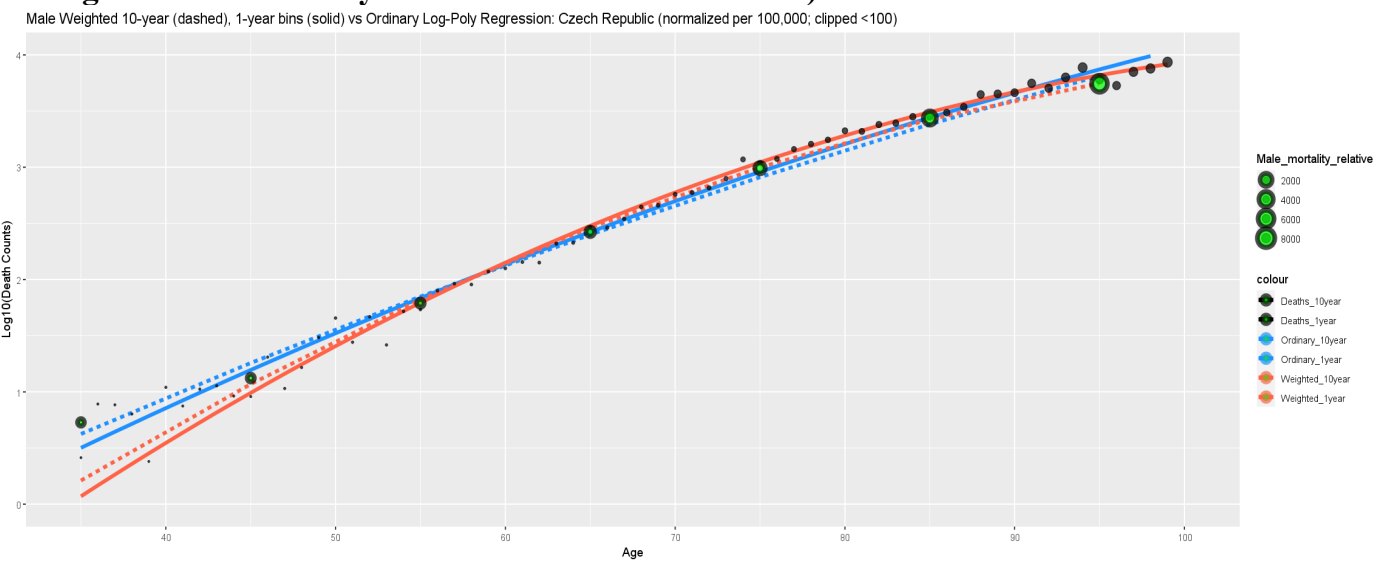

(b)

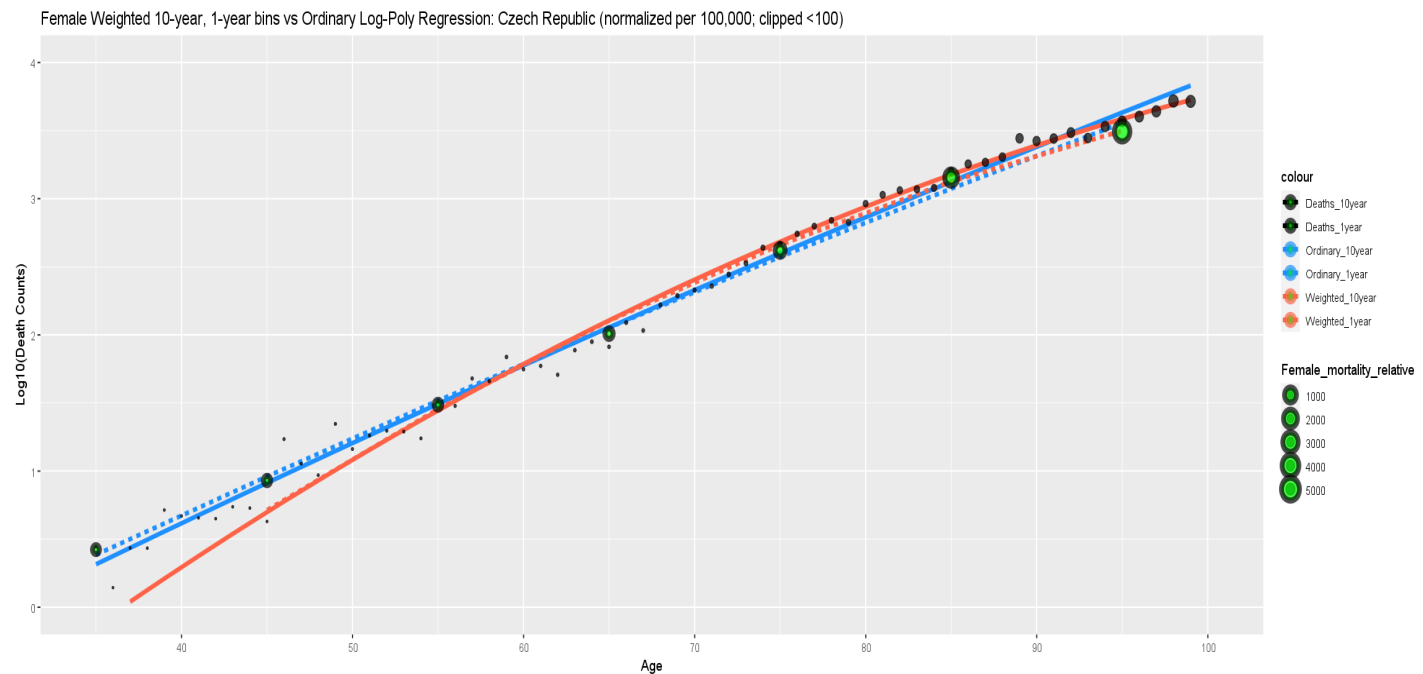

\subsection{Methods: Sex Differential and Age Parity}

The subject of the sex differential (SD), shown in red in Figure 3, in relation to COVID-19 deaths for age gradients has been studied and quantified in e.g. (Guilmoto 2020, Sobotka et al. 2020, Ng et al. 2020, Demombynes 2020). 
Figure 3.

Concepts: (a) COVID-19 mortality sex differential $S D_{a g e}$ and age lag $A L_{a g e} ;(b) S D_{a g e}$ for different ages, including the Age $_{\text {parity }}$ (Age parity).

(a)

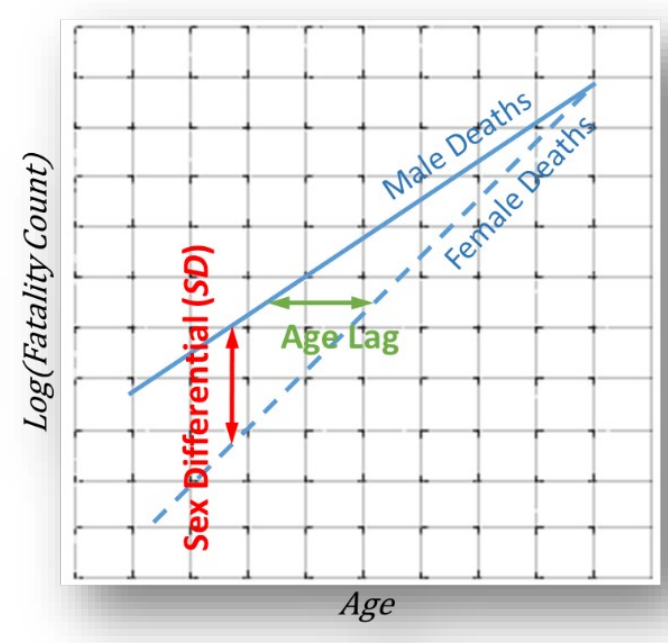

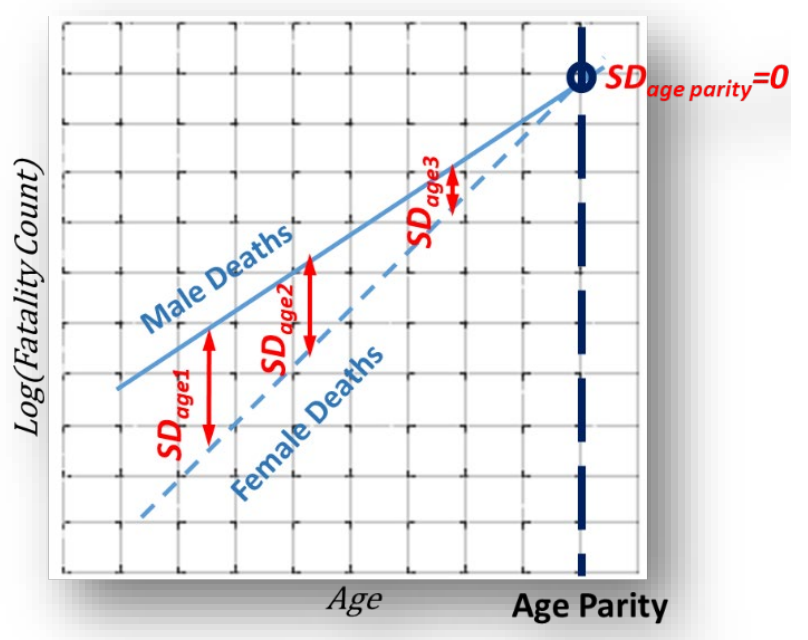

(b)

The log-polynomial regression model from Eq. (1) allows expressing the sex differential $\left(S D_{\text {age }}\right)$ as a $y$ distance between the male and the female $\log \left(F C_{a g e}^{M}\right), \log \left(F C_{a g e}^{F}\right) . F C_{a g e}^{M}$ stands for the Fatality (death) Count-males, and $F C_{a g e}^{F}$ - females, respectively. The idea is illustrated in Figure 3a, where the COVID-19 $S D_{\text {age }}$ is depicted as a vertical gap, shown as a function of age. The formal equation for $S D_{\text {age }}$ is:

$$
\begin{gathered}
S D_{\text {age }}=\log \left(F C_{\text {age }}^{M}\right)-\log \left(F C_{\text {age }}^{F}\right) \text { if measured on the log scale, or } \\
S D_{\text {linear }(\text { age })}=\frac{F C_{a g e}^{M}}{F C_{a g e}^{F}} \text { if measured on the linear scale, }
\end{gathered}
$$

Let us rename $S D_{\text {linear (age) }}$ as $F S R_{\text {age }}$ (Fatality Sex Ratio), often referred to as the M/F ratio in e.g. (Sobotka et al. 2020),

$$
F S R_{\text {age }}=S D_{\text {linear }}(\text { age })
$$

Having regression model fitted (Eq. (1)) to male's and female's fatality count data, $S D_{a g e}$ or $S D_{x}$ can be rewritten to express it analytically, namely:

$$
\begin{gathered}
S D_{x}=\log \left(F C_{x}^{M}\right)-\log \left(F C_{x}^{F}\right)=y^{M}-y^{F}= \\
\left(b_{0}^{M}-b_{0}^{F}\right)+\left(b_{1}^{M}-b_{1}^{F}\right) x+\left(b_{2}^{M}-b_{2}^{F}\right) x^{2}
\end{gathered}
$$

Using Eq. (4) we can predict the age "parity", $A g e_{\text {parity }}$, for the $S D_{x}$, i.e., the age at which the male's odds of dying will be on par with the female's, see Figure $3 \boldsymbol{b}$. For this we need to solve the $\left(b_{0}^{M}-b_{0}^{F}\right)+\left(b_{1}^{M}-b_{1}^{F}\right) x+\left(b_{2}^{M}-b_{2}^{F}\right) x^{2}=0$ equation for $x$, i.e., age in years. The condition of $S D_{x}=0$ will only occur if the female's "advantage" declines at an older age. The results for individual countries are shown in Figure 4 and Table 1. 
Figure 4

Sex differential $S D_{\text {age }}$ and Age parity $A g e_{\text {parity }}$ for various countries. OLS regression is shown with solid, WOLS - with dashed lines.
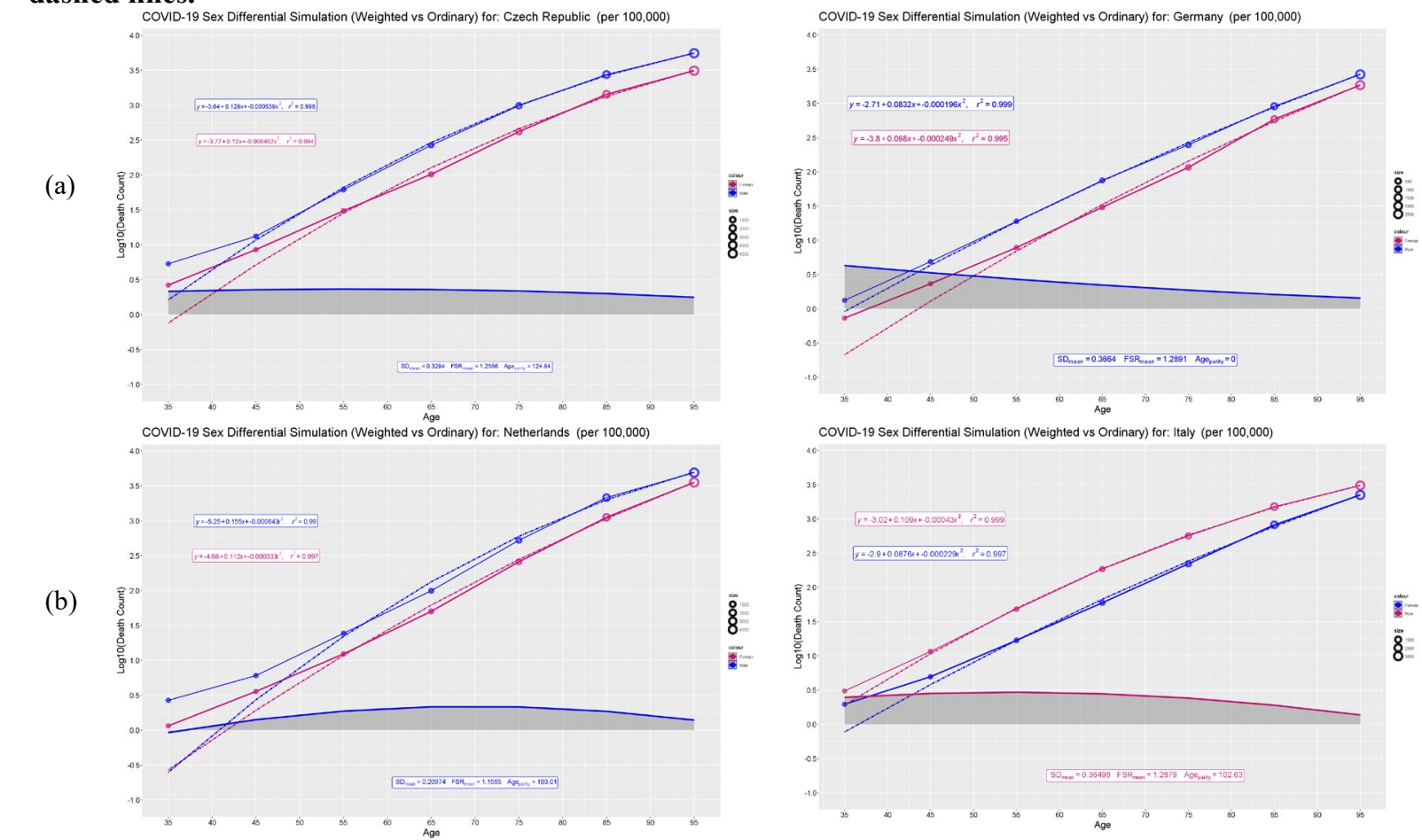

(b)
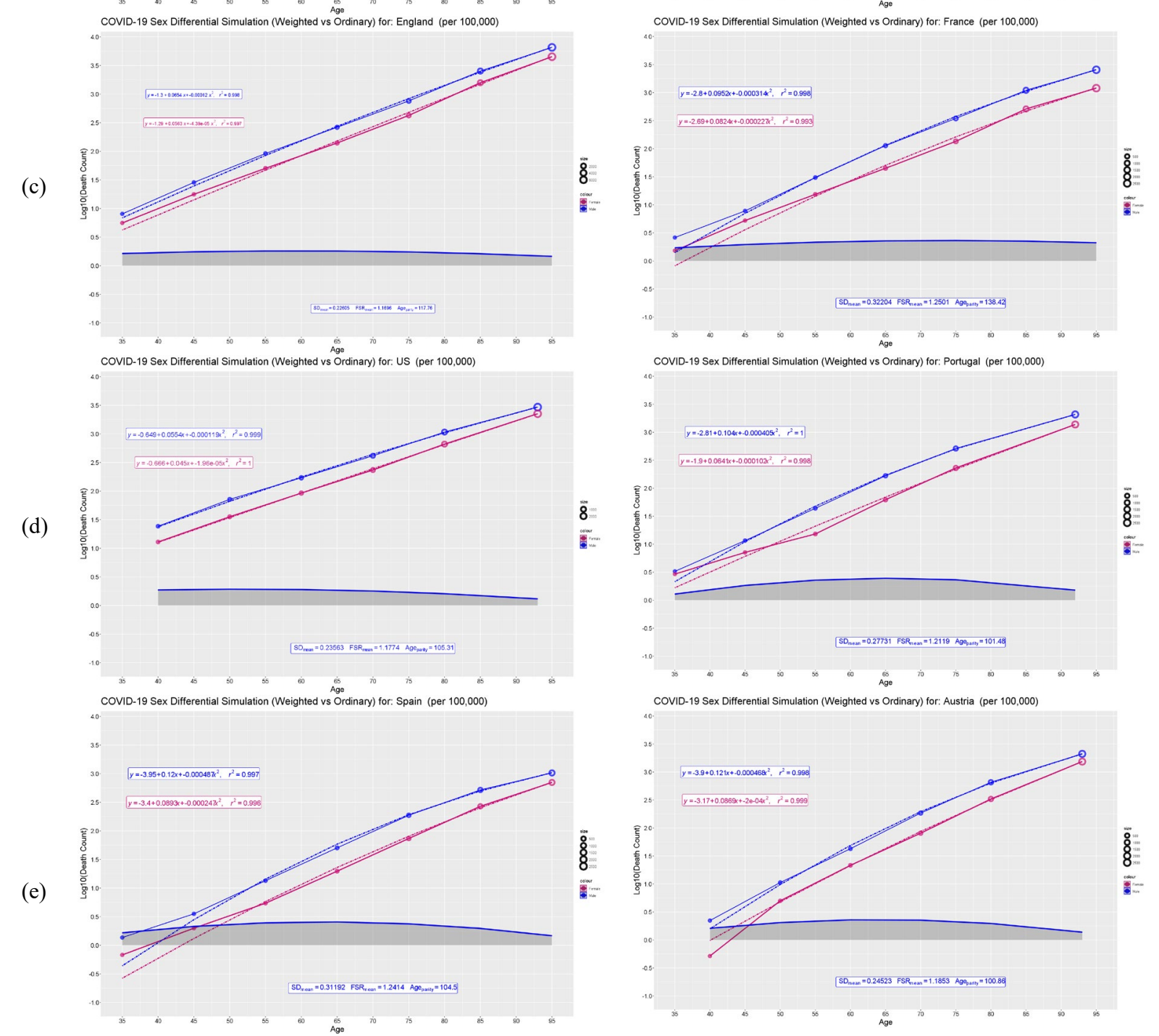
Table 1

Age parity Ag $_{\text {parity }}$ for the ten major countries affected by the COVID-19 pandemic.

\begin{tabular}{|c|c|c|c|c|c|c|c|c|c|c|}
\hline Country & $\begin{array}{l}\text { England } \\
\text { \& Wales }\end{array}$ & Spain & Germany & Italy & France & $\begin{array}{l}\text { Czech } \\
\text { Republic }\end{array}$ & Austria & Portugal & Netherlands & $U S$ \\
\hline$A g e_{\text {parity }}$ & 117.8 & 104.5 & $139 *$ & 102.6 & 138.4 & 124.8 & 100.9 & 101.5 & 103.0 & 105.3 \\
\hline
\end{tabular}

*Note: Germany's female regression polynomial gets as close as $S D_{a g e}=0.04985$ to the male's, but does not reach the parity. See Figure 6 and the discussion there for more on the subject.

\subsubsection{Advantages of Weighted Regression Beyond COVID-19}

Sex-related discrepancies are not unique to COVID-19. Specific sex differentials may vary for different diseases (Mauvais-Jarvis et al. 2020; Harvard Health 2021,). Ng et al. (2020) report ratios of $1.3-2$ for allcause mortalities; compare to 2 - 3 for COVID-19 (Guilmoto 2020). Therefore, scientists will continue researching sex discrepancies with respect to mortality long after the COVID-19 pandemic is over. And fitting regression functions to mortality age gradients will keep on being a useful tool for data modeling and analysis regardless of the death cause. For these reasons getting the regression right and honing the tools is very relevant.

The use of WOLS is justified always whenever the data point (age bin) weights are not uniformly distributed. Many decease' age gradients ("mortality trajectory") or general mortality follows the Gompertz law (Gavrilov and Gavrilova, 2011), which implies a heavy disbalance in weights age-wise. Moreover, the death age distribution of many disease's outbursts is non-uniform either, e.g. the Spanish flu of 1918 (Gagnon 2013), or 2009 pandemic influenza (Karageorgopoulos 2011) with distinct age gradients compared to COVID-19, would all benefit from using the weighted regression instead of the conventional OLS.

\subsection{Methods: Age Lag}

Male fatality COVID-19 deaths (risks) are generally higher than female's (Demombynes 2020; Guilmoto 2020; $\mathrm{Ng}$ et al. 2020). Given a male fatality risk $y_{a g e}^{M}$ and same age female's $y_{a g e}^{F}$, the following holds for most ages: $y_{a g e}^{M}-y_{a g e}^{F}=S D_{a g e}>0$. That is the male's risk of dying from COVID-19 is usually higher than the female's of the same age. How much younger a male should be ( $t$ in years) to "compensate" for the sex differential $S D_{a g e}$, i.e., to equalize the risks: $y_{a g e}^{F}=y_{a g e+t_{a g e}}^{M}$ ? This way a male younger by $t_{a g e}$ will have an equal risk of dying from COVID-19. Therefore, we are referring to this $t_{\text {age }}$ as the "age lag" $A L_{a g e}$, which is depicted in Figure $3 \boldsymbol{a}$ as a horizontal gap shown in green. Age lag shows by how many years $\left(t_{x}\right)$ we need to shift the $x$ ("age") of a male to achieve the risk parity with a female of age $x$. $A L_{a g e}$ denoted as $t_{x}$ in Eq. (5) can be derived from the log-polynomial model analytically at any given age $x$ as:

$$
\begin{gathered}
y_{x}^{F}=y_{x+t_{x}}^{M} \text { or } \\
b_{0}^{F}+b_{1}^{F} x+b_{2}^{F} x^{2}=b_{0}^{M}+b_{1}^{M}\left(x+t_{x}\right)+b_{2}^{M}\left(x+t_{x}\right)^{2},
\end{gathered}
$$

Finally, $A L_{a g e}$, i. e. $t_{a g e}$, is a solution to the quadratic equation for any desirable $x$ (age):

$$
b_{2}^{M} t_{x}^{2}+\left[2 b_{2}^{M} x+b_{1}^{M}\right] t_{x}+\left[\left(b_{2}^{M}-b_{2}^{F}\right) x^{2}+\left(b_{1}^{M}-b_{1}^{F}\right) x+\left(b_{0}^{M}-b_{0}^{F}\right)\right]=0
$$

We can see from Figure 5 that the age lag $A L_{\text {age }}$ typically has a "U"-shape later in life. Interpretation of say $A L_{70}=t_{70}=-10$ is that the odds for the lethal outcome of a 70 years-old female are equal to those of a 60 years-old male. The interpretation of $A L_{a g e}=t_{a g e}=0$ is that males and females of the same age have equal risks of dying from COVID-19. $A L_{\text {age }}=0$ occurs at the age of parity $A g e_{\text {parity }}$. 
Figure 5

Simulated age lag $A L_{a g e}$ for the ten major countries affected by the COVID-19 pandemic.

COVID-19 Age Lag (Weighted): Countries 1-5

(a)

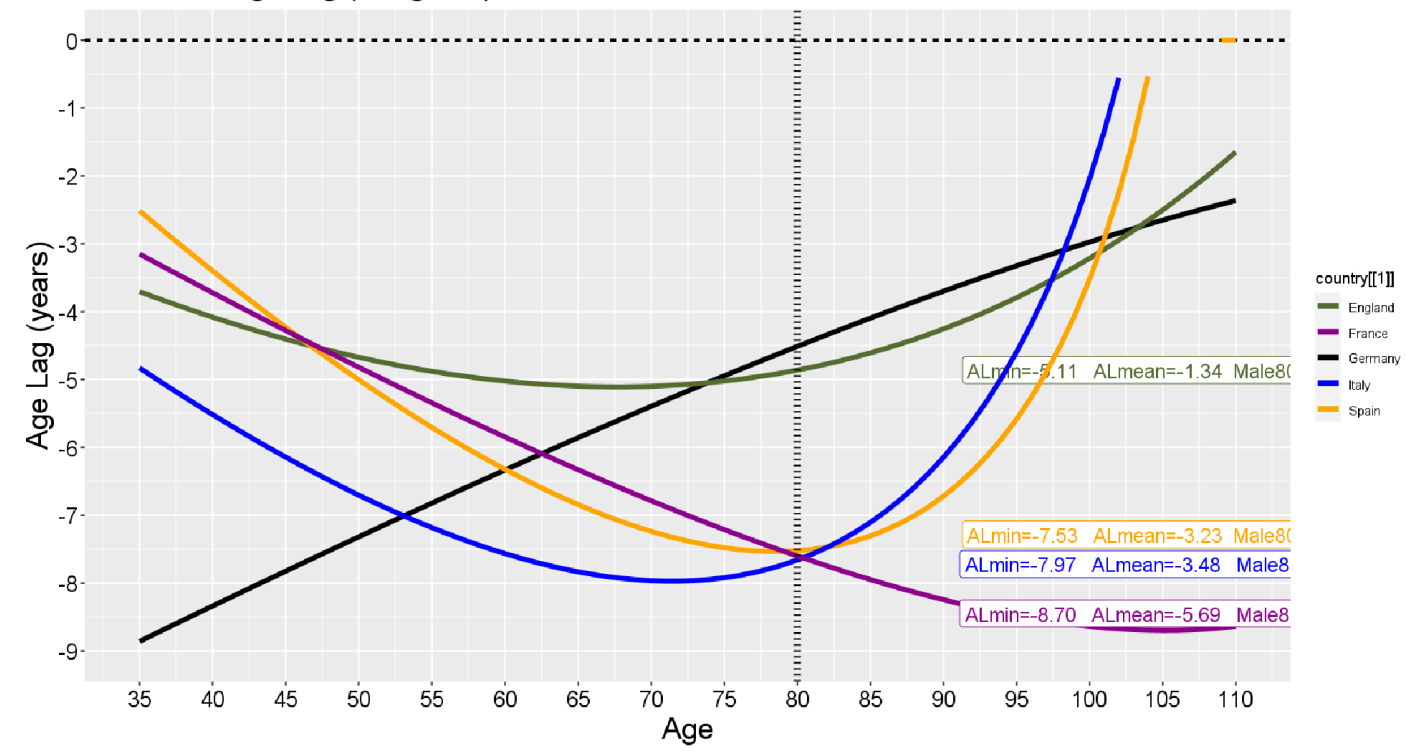

COVID-19 Age Lag (Weighted): Countries 6-10

(b)

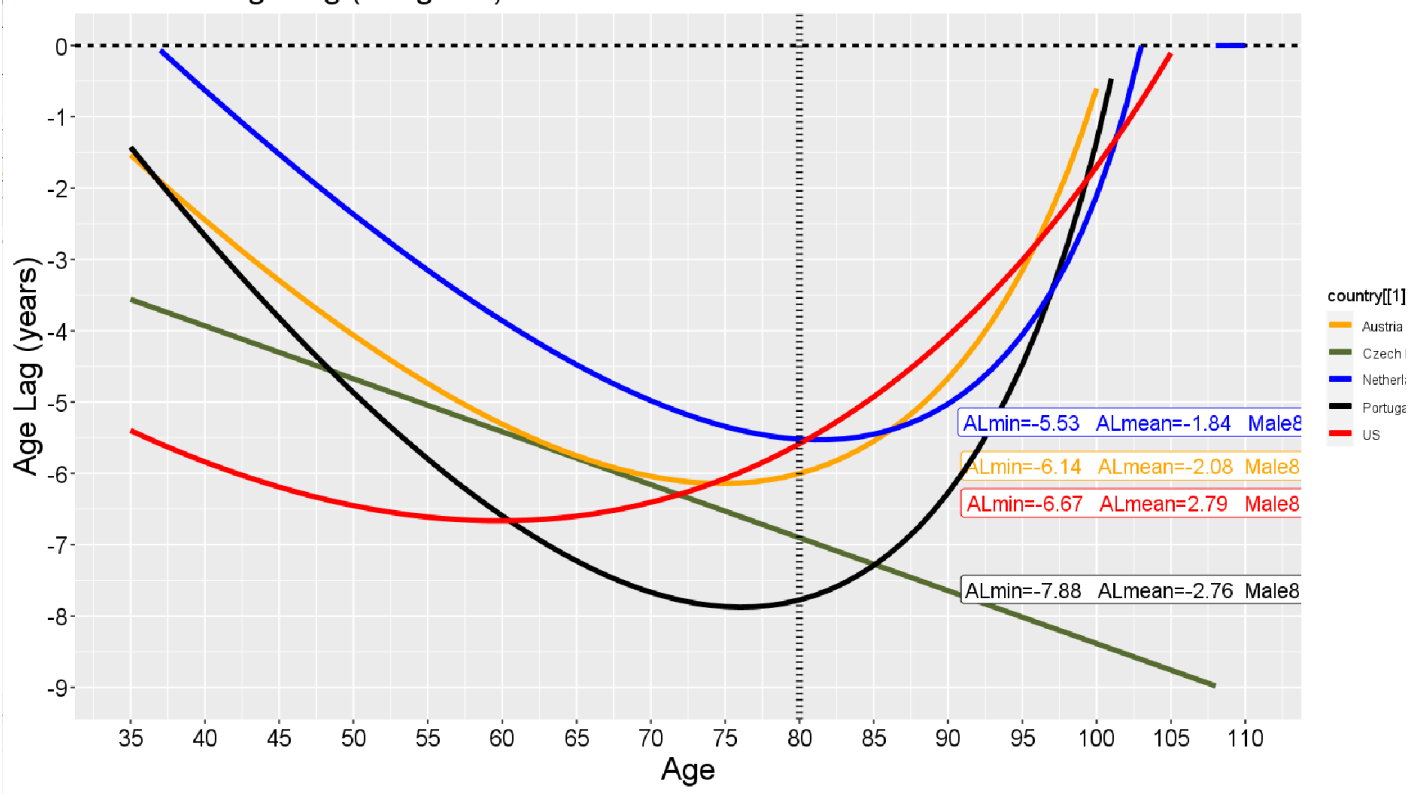

The pattern emerging in Figure 5 shows the maximum negative $A L_{\text {age }}$ to vary in the range of $[-5,-10]$ for a wider interval of ranges from 45 to 80 . After that, the trend reverses: for ages older than 80 the $A L_{\text {age }}$ narrows until reaches 0 around the age of 100 with some exceptions in both directions. This is obviously in line with the $A g e_{\text {parity }}$ that we calculated in Chapter 2.2 and plotted in Figure 4.

The $A L_{\text {age }}$ functions for Germany and the Czech Republic stand out and require a closer look, see Figure 6. In the case of Germany, the female (yellow) regression polynomial is closing in on the male's (black) with age and getting as close $S D_{a g e}=0.04985$ on the logarithmic scale or FSR age (Fatality Sex Ratio, M/F ratio) of 1.12 at the age of 139 but never reaches the $A g e_{\text {parity }}$ exactly. The Czech Republic female's regression polynomial (red) does cross the male's (blue) at just over 124 years, which happens after the male's curve has bent downward. Flatter portions of the curves at ages over 100 years may be less practical from the age lag perspective. A much more robust metric is the parity age ( g $_{\text {parity }}$ ) which takes into account all the data points $\left(A L_{a g e}\right.$ is calculated for every value of age separately, see Figure 6). At the $A g e_{\text {parity }}$, the $A L_{\text {age }}=0$ by definition. 
Table 2

Age Lag $A L_{\text {min }}$ in years for the ten major countries affected by the COVID-19 pandemic.

\begin{tabular}{c|lllllllllll}
\hline Country & $\begin{array}{l}\text { England } \\
\boldsymbol{\&} \text { Wales }\end{array}$ & Spain & Germany & Italy & France & $\begin{array}{l}\text { Czech } \\
\text { Republic }\end{array}$ & Austria & Portugal & Netherlands & US \\
\hline$A L_{\min }$ (years) & -5.11 & -7.53 & $-9.39^{2}$ & -7.97 & -8.7 & $-12.1^{3}$ & -6.14 & -7.9 & -5.53 & -6.67 \\
\hline $\begin{array}{c}A L_{\text {average }} \\
\text { (years) }\end{array}$ & -1.34 & -3.23 & -4.34 & -3.48 & -5.7 & -6.74 & -2.08 & -2.76 & -1.84 & -2.8 \\
\hline
\end{tabular}

Figure 6

Simulated age lag $A L_{a g e}$ for the ten major countries affected by the COVID-19 pandemic.

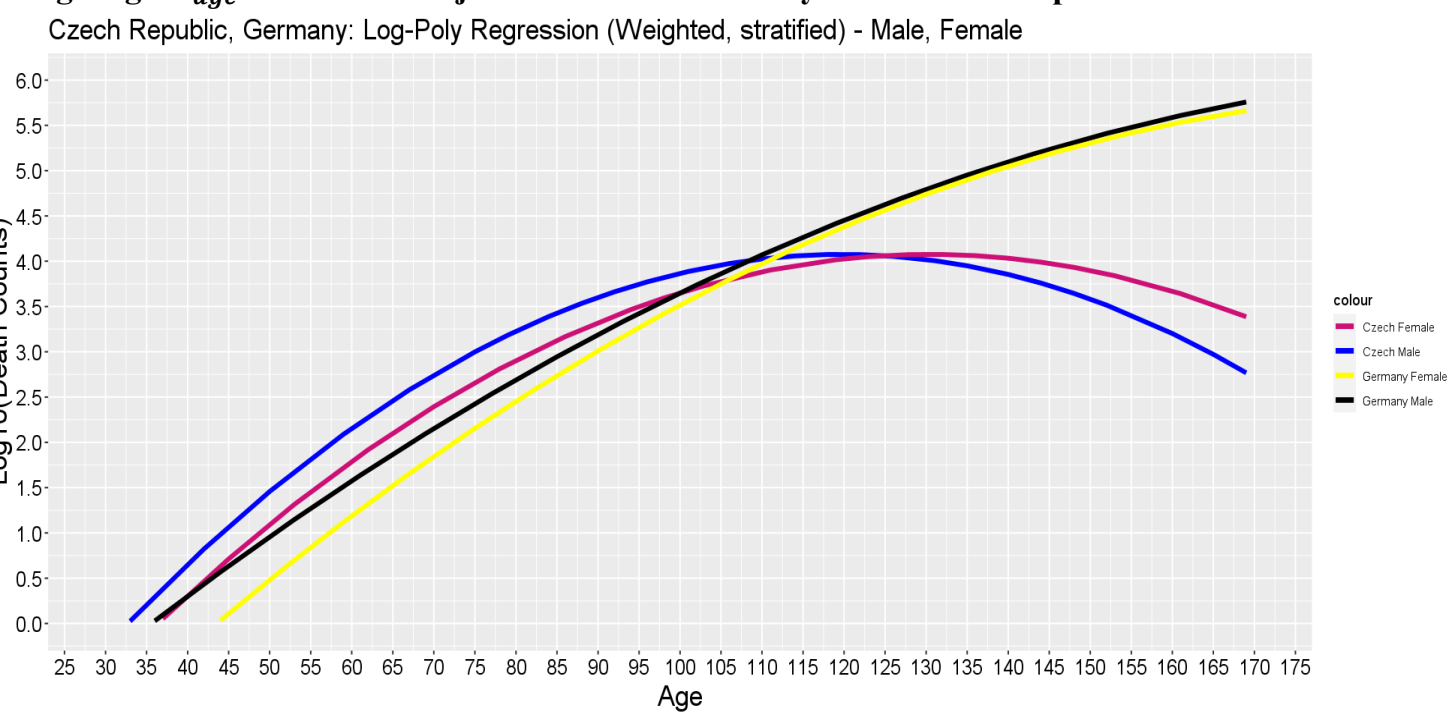

\subsection{Representative Values for Mortality Count Buckets (Bins)}

COVID-19 mortality counts are usually binned into 5- or 10-year ranges in the publicly available age-sex datasets, e.g. (INED 2021). Besides, the half-open oldest age intervals (bins) vary from " $80+$ " to " $100+$ " depending on the country. Likewise, the population data, needed for calculating stratified death counts, typically also comes binned. In absence of more granular data, a single data point, usually the bin center, can be taken as an independent variable (age) estimate or the value representative of that bin (age interval) (INED 2021). For instance, the bin of, say, ages of "60-69", the center of the bin i.e., 65, would be used in fitting a regression polynomial. The author has not found the subject discussed in the literature sources that fitted regression lines to COVID-19 mortality counts. Andersen (2019) used the bin centers, i.e., "age (midpoint of ranges)". Demombynes (2020), Goldstein \& Lee (2020) had not elaborated on the subject explicitly, but if judged by the plots, they used the left boundary of the bin as a representative age value, e.g. "70" for the "70-79" bin and "80" for the half-open interval of " $80+"$ ". A similar approach regarding the last interval is adopted in ( $\mathrm{Ng}$ et al. 2020). We have not found any discussion of whether there is a need for a special attitude towards the half-open bin in the literature. The line of thinking may have been that if the representative age for the half-open interval is consistently taken at the left boundary, e.g., " 80 " for the " $80+$ ", things will work out. The problem here is that it is one thing when " 80 " represents the closed interval of " $80-89$ ", and the other when the same " 80 " represents the half-open of " $80+$ ". For instance, in Germany, $46.6 \%$ (stratified) of COVID-19 deaths belong to the " $80-89$ " age group, but would be $69.8 \%$ if the oldest age bin were " $80+$ ". This is because $23.3 \%$ belong to " $90+$ " (INED 2021); similar proportions can be observed for the Czech Republic in Figure 10(b). All that shows that using "80" as the representative value all across " $80-84$ ", "80-89", or " $80+"$ is problematic. Our simulations in Figure 9 show what kinds of

${ }^{2} A L_{\text {min }}$ is so low Germany because the regressions for male and female for Figure 4 (aright) show the largest sex differential at the early ages, where it is less of interest.

${ }^{3}$ The Czech Republic case is discussed in the subsequent paragraph in more detail. 
distortions it causes.

There are two questions to explore in this chapter:

- For the closed intervals of ages, e.g., "0-9", "10-19", etc.: given the mortality count distribution is non-linear and asymmetric, would the bin center be a reasonable estimate?

- For the open intervals of ages, e.g., "80+", "90+", etc.: what age data point should be used for regression? Several factors would affect the answer. What would be the impact on modeling the data for the countries that report differently structured open intervals?

To help us answer the questions we will refer to the unique (to the author's knowledge) case of the Czech Republic. What makes the latter unique is the fact that it provides access (MZČR Onemocnění Aktuálně 2021) to a very granular COVID-19 mortality data having an exact age at death with a single-year resolution for each sex. This is one part of the equation, we also need the population data to stratify the death counts, and here again the nearly perfectly organized data with a single-year age resolution are available in (Czech Statistical Office 2019) for each sex.

Unfortunately, no granular single-year population data are available for the age over 100 in (Czech Statistical Office 2019) dataset, therefore we excluded the "100" data bucket below. There are only a few dozens of COVID-19 in the Czech Republic in ages over 100 at the time of writing, therefore, we preferred the perfectly stratified data for all ages under 100. This simplifies and streamlines the experiment while keeping the impact minimal. Below, we treat the regression fit to the single-year age resolution age histogram as a virtually "ideal", considering that it is by far the best COVID-19 and population data that can be realistically obtained at this moment. As such it is used as a baseline in our exploration of the binning methods.

The conventional technique of binning data into 5- or 10-year bins instead of the high-resolution raw data may be convenient for multiple reasons, or the only available one as the case of COVID-19 and demographic data which is aggregated by the agencies supplying the data. Exceptions are the half-open intervals for the oldest age. The purpose would be to see how well the centers of the bins would allow fitting the "ideal" full resolution stratified mortality histogram. 
Figure 7

Histogram bin (age group) bucketing: $90+, 80+$ for non-stratified fatality counts. Note the oldest age bin means (in brown) shifting to the right depending on the amount of aggregation.

(a)

Czech Republic Male COVID-19 Deaths Distribution 90+

年)

Czech Republic Male COVID-19 Deaths Distribution 80+

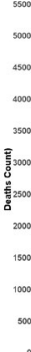

Czech Republic Male COVID-19 Deaths Distribution 90+ (Stratified)

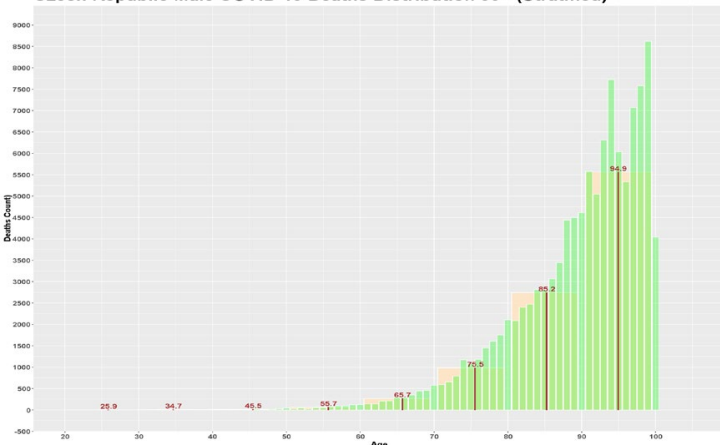

Czech Republic Male COVID-19 Deaths Distribution $80+$ (Stratified)

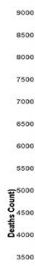

(g)

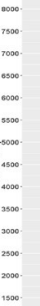

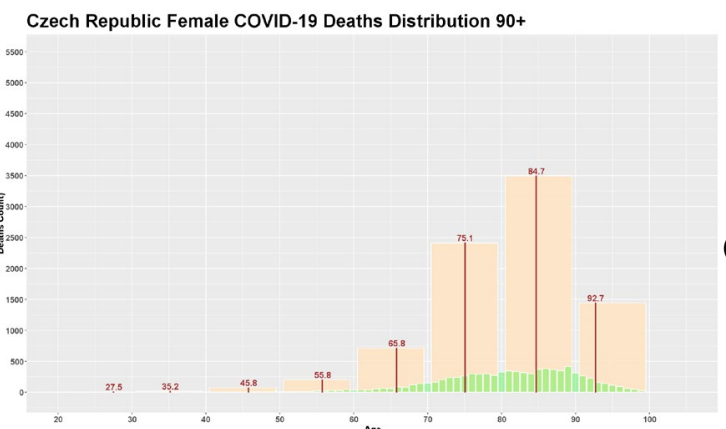

Czech Republic Female COVID-19 Deaths Distribution 80+

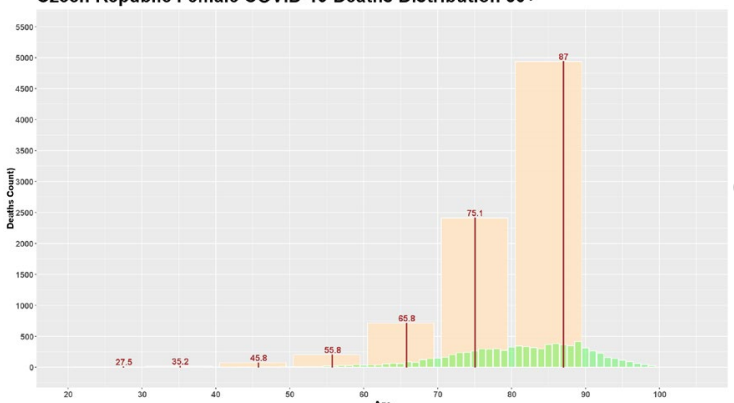

Czech Republic Female COVID-19 Deaths Distribution 90+ (Stratified)

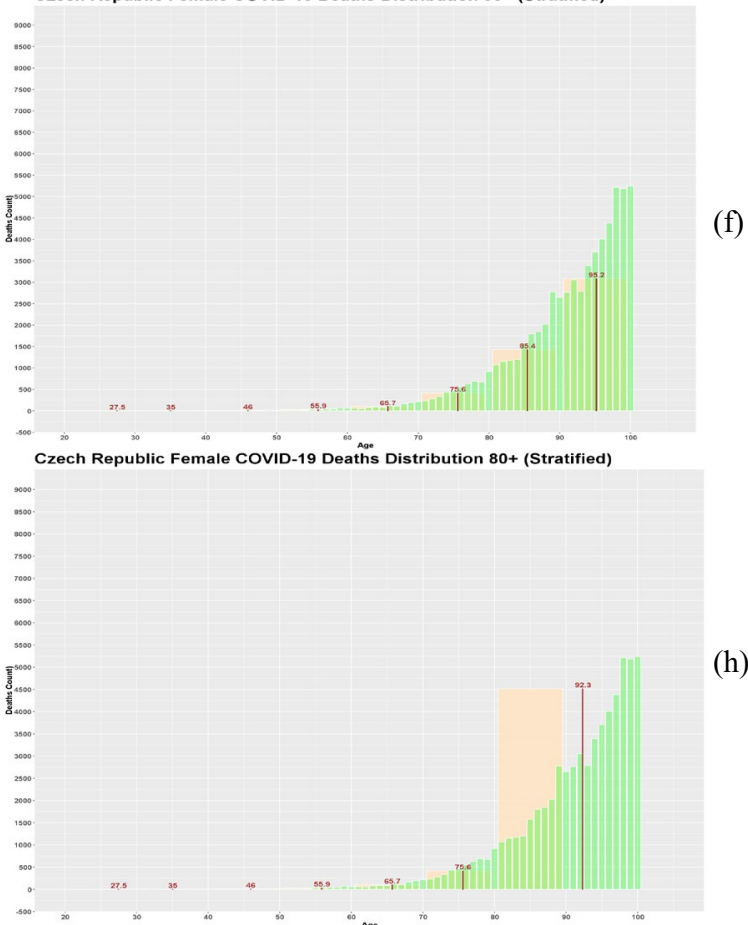

(b)

(d)

(f)

(h) 


\section{Figure 8}

Histogram bin centering: $90+, 80+$ for stratified fatality counts $(\mathrm{Log} 10)$. The beige dashed regression line is a log-poly fit on the bin centers, the brown dashed line is the same for the bin means, shown with relevant regression error RMSE for the full resolution regression (the solid green line).
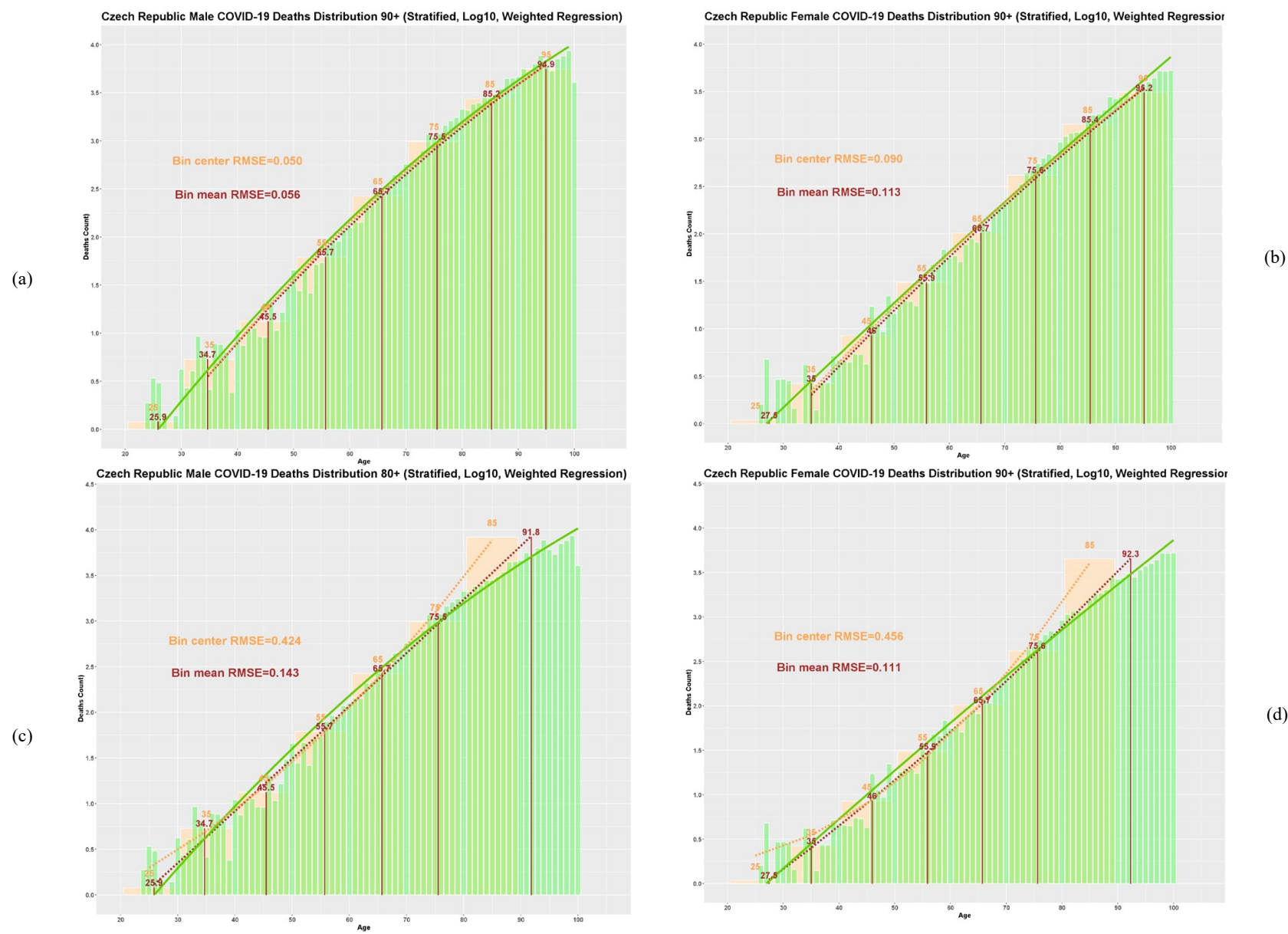

We can see in Figure 7, Figure 8 that centers of the age intervals seem to be representing the means for a given histogram bin reasonably well (the means are displayed with thin brown vertical bars with the values at the top of the bars). We claim that because:

- The mean values are situated very close to the bin centers, see Figure 8 (a-d).

- The regression lines and the RMSEs are reasonably close to each other, which confirms the validity of the conventional approach, see the $90+$ version of the dataset, see Figure $8(a, b)$.

Figure $8(\mathrm{c}-\mathrm{d})$ is another story because of the half-open oldest age interval of $80+$, to be discussed below.

\subsubsection{Half-Open Oldest Age Intervals}

The half-open oldest age intervals of $80+, 90+$ have interval center undetermined. Estimating the mean for it may be possible with the Czech Republic single-year dataset, but not with the binned into 5- or 10-year bin datasets for other countries (INED 2021).

- With no aggregation, i.e., 90+, regression error RMSE of the regression fitted to the centers and the means is a virtual tie, as both regression lines are very close to the ideal fit (shown in beige).

- With more aggressive data aggregation, i.e., $80+$, the issue becomes obvious as the center RMSE skyrockets compared to the RMSE of regression fitted to the means. Apparent reason to it is that the actual mean of the open interval is 91.8 years for male and 92.3 for female, i.e., outside of the 10 year interval of 80-89.

- Note that the actual impact would depend on the population structure of a given country.

\subsubsection{Left Boundary as a Representative Value for Age Intervals}

We mentioned earlier that some authors, e.g. (Demombynes 2020, Ng et al. 2020, Goldstein \& Lee 2020) seemingly used the left boundary of the age interval as a representative value. Let us check the regression 
error compared to the ideal fit, see Figure 9. One can notice much higher regression errors RMSE compared to the bin means, and even to the bin centers from Figure 8 for all levels of aggregation and both sexes. While for $90+$ the left-boundary-based regression manages to track the trend albeit there is a noticeable gap between it and the ideal fit; for $80+$ everything is off including the trend.

\section{Figure 9}

Histogram bin centering: $90+, 80+$ for stratified fatality counts $(\log 10)$. Weighted log-poly fit regression to the original fullresolution data is shown with the solid green line. The beige regression line fits the bin left boundaries, the brown line is the fit to the bin means, shown with relevant RMSE for the full-resolution regression.

(a)

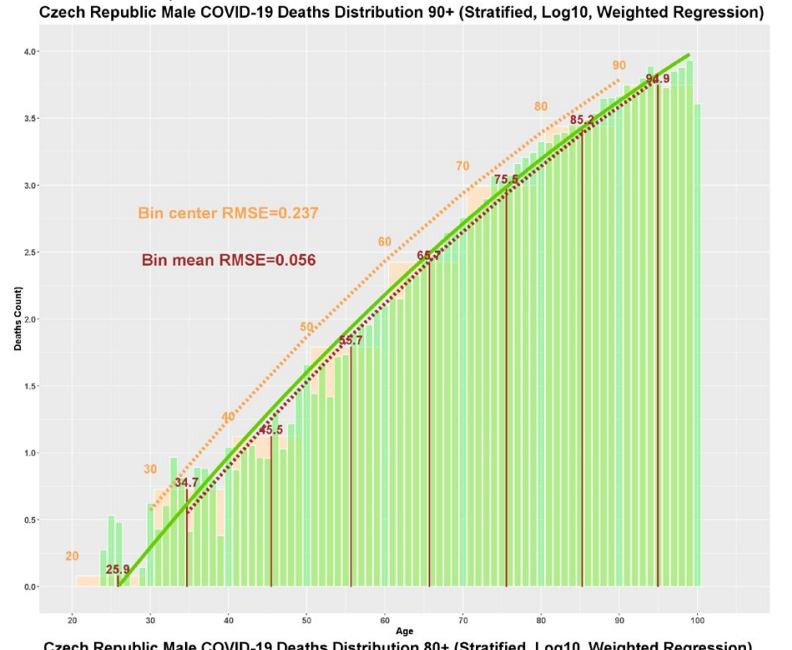

(c)

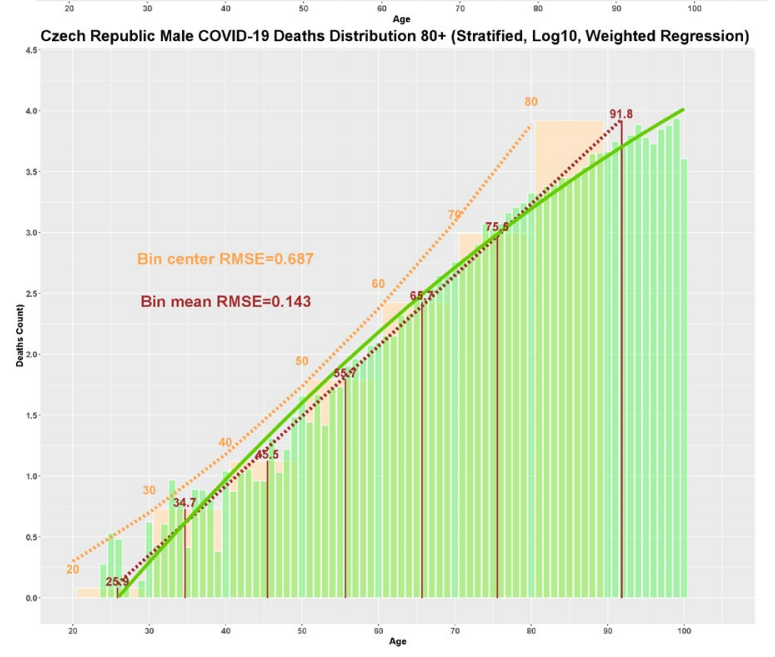

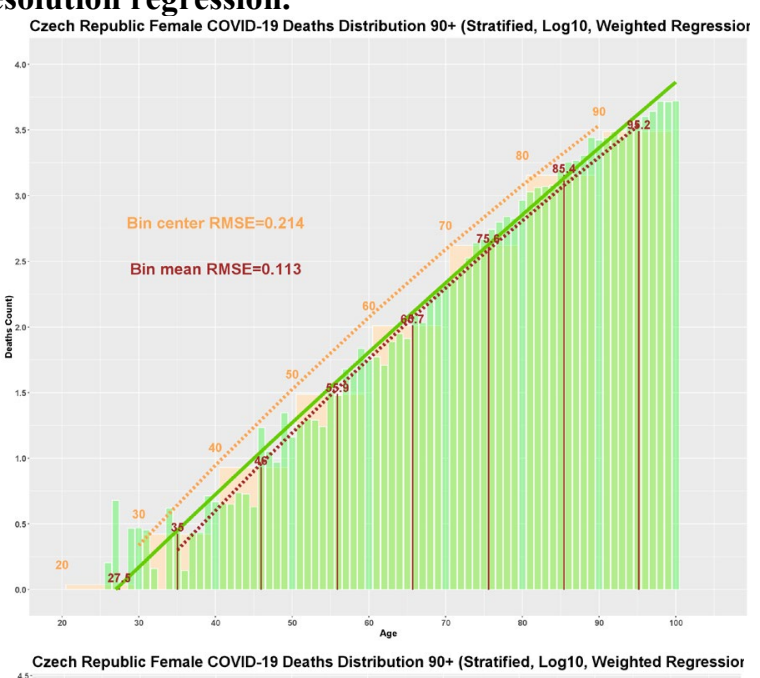

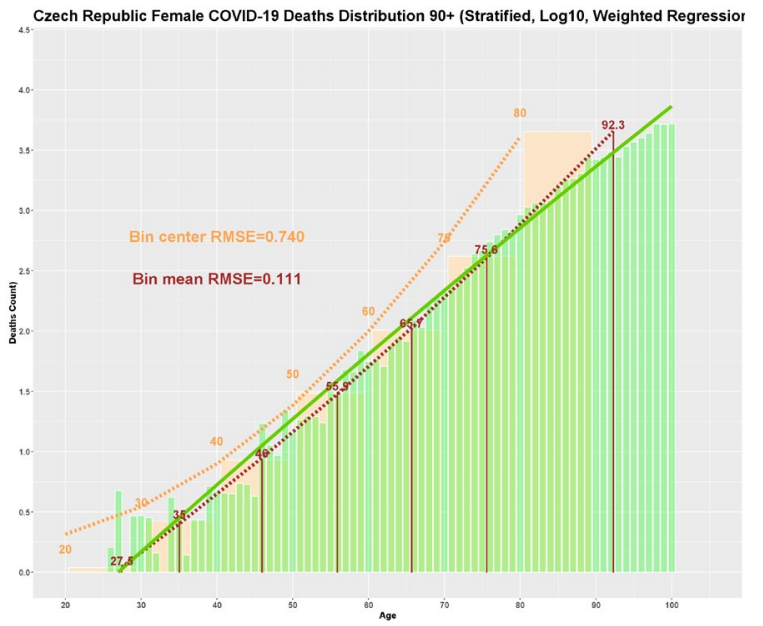

(b)

(d)

\subsubsection{Recommendations for Selection of the Representative Value for Age Intervals}

There are two recommendations from the analysis above:

1. When fitting regression to age-sex mortality use either center or means of the age bins if the means are available.

2. For the half-open interval for the oldest age use the mean if the assessment of such is possible. In absence of the latter, use the means calculated for the Czech Republic in Table 3.

Mean locations depend on the bucketing of the oldest age death counts into the half-open interval. Table 3 has the means calculated for the Czech Republic simulations with various half-open intervals. They depend on the population structure of the country, as well as on the COVID-19 death age-sex structure, see e.g., these for the Czech Republic in Figure 10. The most surprising finding is that the means for 80+ and 95+ intervals are situated much further away from the expected ones.

In our experiments, we have applied values from the last row in Table 3 for both sexes for all country datasets, which implicitly assumes similarity with the Czech Republic. The latter assumption relies on the fact that the Czech Republic profile is similar to many of the observed countries, as it belongs to the same demographic cluster, situated in the same geographic region, etc. Besides, this was the only option that was supported by the data. Further research is needed here, and having more granular datasets from the other nation's authorities would help in the process tremendously. 
Table 3

Oldest age half-open interval means (the original dataset was censored to $<=99$ years).

\begin{tabular}{|c|c|c|c|c|}
\hline Oldest Age Interval & $95+$ & $90+$ & $85+$ & $80+$ \\
\hline Female Age Mean & 97.21 & 94.93 & 93.23 & 91.83 \\
\hline Male Age Mean & 97.18 & 95.18 & 93.5 & 92.3 \\
\hline Used in the experiments - both sexes & NA & 95 & 93 & 92 \\
\hline
\end{tabular}

Figure 10

The Czech Republic: Population pyramid and age-sex COVID-19 mortality structure.

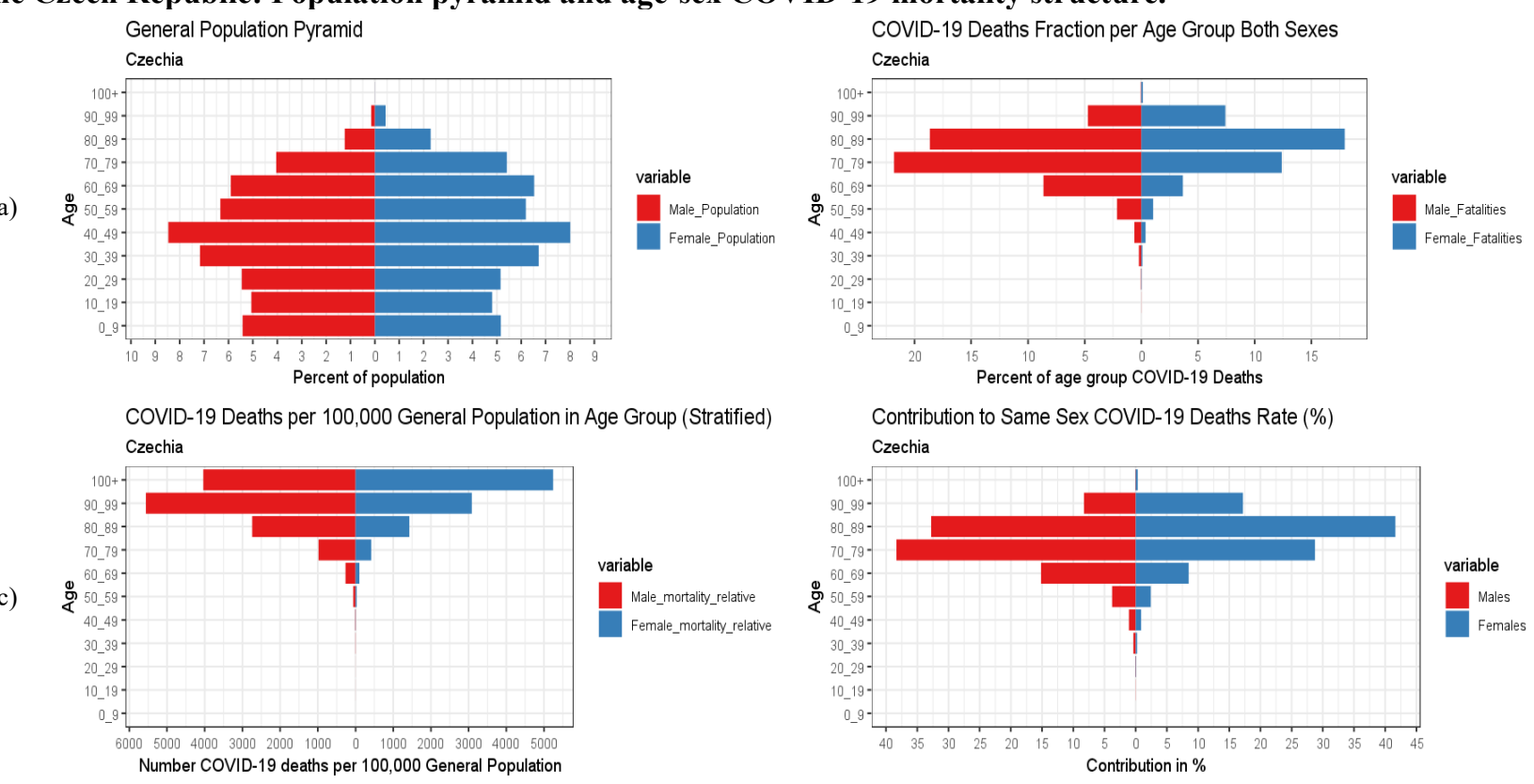

(b)

\section{A Use Case of the Age Lag: COVID-19 Vaccine Prioritization}

Many countries, states, and municipalities are actively rolling out public vaccination campaigns these days. An important question is how to prioritize different strata of the population to minimize the risks to the public as a whole. Ferranna et al (2021) compiled a table of various goals stated by government institutions that are in charge of setting policies and priorities for vaccine allocation in individual countries (US, UK) and worldwide (WHO). Such goals vary from the more obvious 'reduce mortality" to the less e.g., "reduce negative societal impact, mitigate of health inequities, treat people fairly, minimize impact". Based on the goals stated above certain countries would choose to prioritize higher certain occupations, e.g., high-risk health workers and first responders, personnel in long-term facilities, racial or ethnical minority status of population groups, their socioeconomical vulnerability etc., (Ferranna et al, 2021). Out of all possible considerations our approach aims at minimizing the overall mortality based on age and sex, while respecting other considerations and leaving it up to the policymakers to apply our recommendations selectively.

Age-based approach has been incorporated into virtually all known prioritization schemes, namely:

- Three age-based tiers in the US: "75+", "65-74”, "16-64" for all the sexes (CDC 2021). Many US states are implementing more granular age cutoffs.

- Four in Germany: "80+", "70-79”, "60-69”, “<60" (Cylus 2021)

- Eight age-based tiers in the UK: "80+", “75-79”, "70-74”, “65-69”, “60-64”, "55-59”, "50-54”, " $<50$ " (Priority Groups 2020, Ferranna et al, 2021).

- "Israel's initial age cut-off of 60 years and over... Estonia focuses first on those 70 years and over" (Cylus 2021). 
A general remark to all the reasoning in this chapter. We are assuming no differences between the sexes in all other aspects that may affect COVID-19 mortality, like comorbidities, race, origin, occupation, virus load, etc.

Regardless of the small variations among individual countries, the age-based "unisex" policy dominated the risk-based vaccination priority. World Health Organization (WHO SAGE 2020) acknowledging that "...there is evidence that the risk of severe disease and death is higher in males than in females, particularly in older age groups" in "Gender Considerations" chapter advised against prioritization based on gender to not "...exacerbate underlying gender-based inequities". Bubar et al (2021) mentioned sex among other factors affecting COVID-19 mortality, but have not included it in their model. There is an obvious contradiction here; higher prioritization of the older ages would undoubtedly reduce the overall risk, and ignoring sex-related risk discrepancy is a step in the wrong direction. The latter conclusion becomes obvious after we got to understand how the sex discrepancy directly translates into the age lag, see Figure 3: ignoring the male sex for prioritization is equivalent to ignoring age differences of say 5-10 years. And ignoring the age differences for the same se goes against the consensus; see Table 2 and Figure 5 for specific values of the age lag. The truly optimal strategy would be to incorporate the sex factor in the risk assessment; this way males would have been prioritized higher than the females of equal ages. We can see that this might have been a tough sale from the political perspective, especially when the subject of gender equity/equality is near the top of the public agenda. Advocating "preferences" for males is challenging in such an environment, but it would save lives.

From the pure risk perspective, we could use the Age Lag methodology from Chapter 2.3 to answer questions like e.g. "what is the male's age at which his risk of dying from COVID-19 is equal to female's at 80 ?". To calculate it one should plug $x=80$ into Eq. (6) to obtain $A L_{80}$. Instead of 80, any countryspecific age cut-off (set of cut-offs) could be used, ideally derived from the COVID-19 mortality statistics for this given country. Namely, the Male Equal Risk for the age of 80 , i.e., $M E R_{80}$, can be calculated through $A L_{\text {age }}$ as:

$$
M E R_{80}=80+A L_{80},
$$

or directly by solving the quadratic equation with respect to $M E R_{80}$ in:

$$
b_{0}^{F}+b_{1}^{F} 80+b_{2}^{F} 80^{2}=b_{0}^{M}+b_{1}^{M} M E R_{80}+b_{2}^{M} M E R_{80}^{2}
$$

Any specific age can be easily plugged in Eq. (7).

Table 4

Male Equal Risk for the age of 80 for the ten major countries affected by the COVID-19 pandemic.

\begin{tabular}{l|llllllllll}
\hline Country & $\begin{array}{l}\text { England } \\
\text { \& Wales }\end{array}$ & Spain & Germany & Italy & France & $\begin{array}{l}\text { Czech } \\
\text { Republic }\end{array}$ & Austria & Portugal & Netherlands & US \\
\hline$M E R_{80}$ & 75.1 & 72.5 & 75.4 & 72.3 & 72.5 & 73.17 & 73.9 & 72.2 & 74.5 & 74.3 \\
\hline
\end{tabular}

Making the males of ages between these shown in Table 4 wait, instead of prioritizing them along with the females the age of 80 , increases the mortality risks for the individuals. This applies to all age groups and will result in unwarranted losses of lives.

Table 5

Male Equal Risk for various typical cutoff ages for the select countries (USA, Austria) for the sake the risk-based COVID-19 vaccine prioritization.

\begin{tabular}{c|lllllllll}
\hline \multicolumn{1}{c}{ Cutoff Age } & $\mathbf{9 0}$ & $\mathbf{8 5}$ & $\mathbf{8 0}$ & $\mathbf{7 5}$ & $\mathbf{7 0}$ & $\mathbf{6 5}$ & $\mathbf{6 0}$ & $\mathbf{5 5}$ & $\mathbf{5 0}$ \\
\hline USA: $M E R_{\text {cutoff }}$ & 85.7 & 79.9 & 74.3 & 68.8 & 63.5 & 58.4 & 53.3 & 48.4 & 43.6 \\
\hline Austria: $M E R_{\text {cutoff }}$ & 85.1 & 79.3 & 73.9 & 68.9 & 64 & 59.3 & 54.8 & 50.4 & 46.1 \\
\hline
\end{tabular}

An ideal risk-based vaccination campaign would assess in which arm each given dose should go to minimize the overall risk. Since this is not quite practical on the scale, the age-based tiers can be retained but 
adjustments that would take into consideration the sex-age factors are necessary.

A typical COVID-19 age-based unisex vaccination campaign would typically roll out as a sequence of the age-cutoff phases that would decline by 5 or 10 years at each phase (Ferranna et al, 2021, Priority Groups 2020, Cylus 2021). One such possible sequence of phases is shown in Table 5. If we were to incorporate the sex factor, one sex-age mortality risk-based vaccination phase $(i)$ would look like that shown in Figure 11. At this phase- $I$, one of the cutoff ages from e.g. Table 5 , is applied.

The conventional unisex prioritization approach carries excessive risks. One such risk zone is shown as the hashed zone in Figure 11, this zone is excluded in the proposed sex-age-based approach and the lives of the individuals from that zone will be likely spared unlike with the unisex approach. It would be possible to estimate the lifesaving potential as an integral across all phases (cutoffs) in a vaccination campaign. Vaccine efficacy would be a coefficient to the integral.

Figure 11.

The rollout of the COVID-19 sex-age mortality risk-based vaccination campaign versus the conventional "unisex" one, is shown for phase-i.

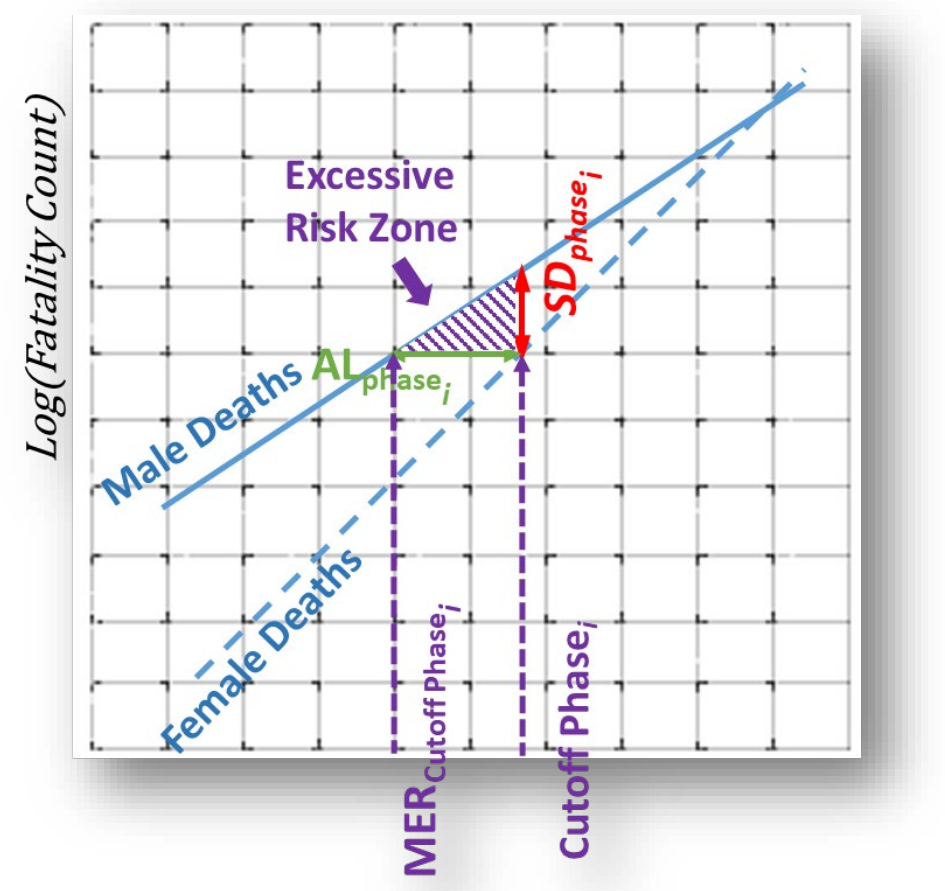

\section{Conclusion}

COVID-19 mortality risks are strongly affected by sex and age proportions in a given population. To concisely describe them analytically and facilitate dissecting trends, many scientists are fitting straight regression lines using the OLS (Ordinary Least Squares) method to the logarithms of the death counts. The paper has defined two goals and delivered on:

- Improve/refine the regression fitting methodology by:

- Apply a more sensitive log-polynomial regression model that is more suitable to the data at hand.

- Apply OWS (Ordinary Weighted Least Squares) when fitting regression to accommodate for non-even distribution of weights along with the age groups.

- Analyze the subject of the selection of representative values for death count intervals (bins)

- Introduce or further refine useful metrics that build upon the above-mentioned methodology

- Age parity, $A g e_{\text {parity }}$, i.e., the age (in years) at which both sexes are equally vulnerable to the COVID-19.

○ Age lag, $A L_{\text {age }}$, (in years) to establish parity between male's and female's risks. 
- Male Equal Risk, $M E R_{\text {female_age, }}$, male's age (in years) at which the risk is equal to female's at female_age. MER approach allows solving such practical problems as mortality risk prioritization of COVID-19 vaccine based on age-sex factors. A scheme of how to quantify the expected lives saved, if such approach is adopted, is provided.

The results based on the analysis of 900,000 COVID-19 deaths from ten countries, heavily affected by the pandemic, show that the well-established trend of male's higher vulnerability persists for most ages. However, with help of modeling, we can see how the trend changes and female's vulnerability is getting higher at very old, often unrealistic, age; the explanation of which is yet to be proposed. The modeling also helps in decision making when prioritizing COVID-19 vaccines administrated to the population, based on sex and age ensuring sound risk-based policy.

Virtually all the questions and data modeling methodologies discussed above are not at all specific to the COVID-19 and can apply to analyzing the sex-age aspect of other diseases.

\section{References}

Anderson, C., 2019. “Poisson Regression for Regression of Counts and Rates - Edps/Psych/Soc 589,”. University of Illinois, https://education.illinois.edu/docs/default-source/carolynanderson/edpsy589/lectures/4 glm/4glm 3 beamer_post.pdf

Atkinson, E., Crowson, S., Pedersen, R., and Therneau, T. "Poisson Models for Person-Years and Expected Rates," Mayo Clinic, Technical Report \#81, September 3, 2008. https://www.mayo.edu/research/documents/biostat$81 \mathrm{pdf} /$ doc-10026981

Bubar, K., Reinholt, K., Kissler, S., Lipsitch, M., Cobey, S., Grad, Y., and Larremore, D. 2021. "Model-Informed COVID-19 Vaccine Prioritization Strategies by Age and Serostatus.” Science 371 (6532): 916-21. https://doi.org/10.1126/science.abe6959.

CDC. 2021. "How CDC Is Making COVID-19 Vaccine Recommendations." Centers for Disease Control and Prevention. March 3, 2021. https://www.cdc.gov/coronavirus/2019-ncov/vaccines/recommendations-process.html.

Cheng, Y., Gregg, E., Rolka, D., and Thompson, T. 2016. "Using Multi-Year National Survey Cohorts for Period Estimates: An Application of Weighted Discrete Poisson Regression for Assessing Annual National Mortality in US Adults with and without Diabetes, 2000-2006.” Population Health Metrics 14 (December). https://doi.org/10.1186/s12963-016-0117-x.

Cylus, J., Panteli, D., and van Ginneken, E.. 2021. "Who Should Be Vaccinated First? Comparing Vaccine Prioritization Strategies in Israel and European Countries Using the Covid-19 Health System Response Monitor." Israel Journal of Health Policy Research 10 (1): 16. https://doi.org/10.1186/s13584-021-00453-1.

Currie, I. 2013. "Fitting Models of Mortality with Generalized Linear and Non-Linear Models.," Technical Report, http://www.macs.hw.ac.uk/ iain/research/Paper_2013.pdf

Czech Statistical Office. n.d. Population by sex and age as at 31.12.2019, Accessed March 8, 2021. https://vdb.czso.cz/vdbvo2/faces/en/index.jsf?page=vystup-objektvyhledavani\&vyhltext $=$ sex\&bkvt $=\mathrm{c} 2 \mathrm{~V} 4 \& \mathrm{katalog}=$ all\&pvo=DEMD001.

Demombynes, G. 2020. COVID-19 Age-Mortality Curves Are Flatter in Developing Countries. Policy Research Working Paper;No. 9313. World Bank, Washington, DC. (c) World Bank. https://openknowledge.worldbank.org/handle/10986/34028 License: CC BY 3.0 IGO.

Ferranna, M., Cadarette, D., and Bloom, D. 2021. "COVID-19 Vaccine Allocation: Modeling Health Outcomes and Equity Implications of Alternative Strategies.” Engineering, May. https://doi.org/10.1016/j.eng.2021.03.014.

Gagnon, A., Miller, M., Hallman, S., Bourbeau, R., Herring, D., Earn, D., and Madrenas, J. 2013. "Age-Specific Mortality During the 1918 Influenza Pandemic: Unravelling the Mystery of High Young Adult Mortality." PLoS ONE 8 (8). https://doi.org/10.1371/journal.pone.0069586.

Gavrilov, L. and Gavrilova, N. 2011. Mortality Measurement at Advanced Ages. North American Actuarial Journal 15 (3): 432-47. https://doi.org/10.1080/10920277.2011.10597629.

Goldstein, J., and Lee, R. 2020. "Demographic Perspectives on the Mortality of COVID-19 and Other Epidemics." Proceedings of the National Academy of Sciences, August. https://doi.org/10.1073/pnas.2006392117.

Guilmoto, C. 2020, COVID-19 death rates by age and sex and the resulting mortality vulnerability of countries and regions in the world, medRxiv 2020.05.17.20097410; https://doi.org/10.1101/2020.05.17.20097410. Accessed 25 July, 2020

Harvard Health. n.d. "Mars vs. Venus: The Gender Gap in Health.” Harvard Health. Accessed March 13, 2021. https://www.health.harvard.edu/newsletter_article/mars-vs-venus-the-gender-gap-in-health. 
INED 2021., The Demography of COVID-19 Deaths (2021). National Institute for Demographic Studies (INED) (distributor), https://dc-covid.site.ined.fr/en/data, Accessed March 3, 2021.

Kala, A., Tiwari, C., Mikler, A., and Atkinson, S. 2017. "A Comparison of Least Squares Regression and Geographically Weighted Regression Modeling of West Nile Virus Risk Based on Environmental Parameters." PeerJ 5 (March). https://doi.org/10.7717/peerj.3070.

Karageorgopoulos, D., Vouloumanou, E., Korbila, I., Kapaskelis, A., and Falagas, M. 2011. "Age Distribution of Cases of 2009 (H1N1) Pandemic Influenza in Comparison with Seasonal Influenza." PLoS ONE 6 (7). https://doi.org/10.1371/journal.pone.0021690.

Mauvais-Jarvis, F., Merz, N., Barnes, P., Brinton, R., Carrero, J-J.,DeMeo, D., De Vries, G.,et al. 2020. “Sex and Gender: Modifiers of Health, Disease, and Medicine.” The Lancet 396 (10250): 565-82. https://doi.org/10.1016/S0140-6736(20)31561-0.

MZČR Onemocnění Aktuálně 2021. COVID-19 v ČR: Otevřené datové sady a sady ke stažení, n.d. onemocneniaktualne.mzcr.cz. Accessed January 3, 2021. https://onemocneni-aktualne.mzcr.cz/api/v2/covid-19. -> Accessed February 28, 2021. https://onemocneni-aktualne.mzcr.cz/api/v2/covid-19 COVID-19: Přehled úmrtí dle hlášení krajských hygienických stanic (umrti.csv)

Ng, J., Bakrania, K., Russell, R. and Falkous, C. 2020. COVID-19 Mortality Rates by Age and Gender: Why Is the Disease Killing More Men than Women? July 3, 2020. https://www.rgare.com/knowledgecenter/media/research/covid-19-mortality-rates-by-age-and-gender-why-is-the-disease-killing-more-men-thanwomen, Accessed January 3, 2021

"Priority Groups for Coronavirus (COVID-19) Vaccination: Advice from the JCVI, 2 December 2020." n.d. GOV.UK. Accessed March 13, 2021. https:/www.gov.uk/government/publications/priority-groups-forcoronavirus-covid-19-vaccination-advice-from-the-jcvi-2-december-2020/priority-groups-for-coronavirus-covid19 -vaccination-advice-from-the-jcvi-2-december-2020.

Shapiro, V. 2020, Modelling COVID-19 age-sex mortality metrics, Wittgenstein Centre Conference 2020 Demographic Aspects Of The Covid-19 Pandemic And Its Consequences,

https://www.oeaw.ac.at/fileadmin/subsites/Institute/VID/PDF/Conferences/2020/COVID19/Posters/1.3_shapiro.pdf, Accessed 13 March 2021.

Shin, Hee-Choon. 2013. Weighted Least Squares Estimation with Sampling Weights, JSM - Survey Research Method Section, 1523-1530

Sobotka, T., Brzozowska, Z., Muttarak, R., Zeman, K. and Di Lego, V. 2020. Age, gender and COVID-19 infections, medRxiv 2020.05.24.20111765; https://doi.org/10.1101/2020.05.24.20111765

Tai, T-H, and Noymer, A. 2018. "Models for Estimating Empirical Gompertz Mortality: With an Application to Evolution of the Gompertzian Slope." Population Ecology 60 (March). https://doi.org/10.1007/s10144-018-0609$\underline{6}$.

Wang, Y., Hao, H., and Wu, C. 2020. "Forecasting Stock Returns: A Time-Dependent Weighted Least Squares Approach." Journal of Financial Markets, May, 100568. https://doi.org/10.1016/j.finmar.2020.100568.

"WHO SAGE Roadmap For Prioritizing Uses Of COVID-19 Vaccines In The Context Of Limited Supply.", November 2020, n.d. Accessed March 26, 2021. https://www.who.int/publications/m/item/who-sage-roadmapfor-prioritizing-uses-of-covid-19-vaccines-in-the-context-of-limited-supply.

Willett, J. and Singer, J. 1988. Another Cautionary Note about R2: Its Use in Weighted Least-Squares Regression Analysis. The American Statistician 42 (3): 236-38. https://doi.org/10.1080/00031305.1988.10475573. 